\title{
Laws of reflection and Snell's law revisited by video modeling
}

\section{Rodrigues, P. Simeão Carvalho}

M. Rodrigues, P. Simeão Carvalho, "Laws of reflection and Snell's law revisited by video modeling," Proc. SPIE 9289, 12th Education and Training in Optics and Photonics Conference, 928922 (17 July 2014); doi: $10.1117 / 12.2070784$

SPIE Event: 12th Education and Training in Optics and Photonics Conference, 2013, Porto, Portugal 


\title{
Laws of Reflection and Snell's Law revisited by Video Modeling
}

\author{
M. Rodrigues ${ }^{\text {a,b }}$, P. Simeão Carvalho ${ }^{\text {b,c }}$ \\ ${ }^{a}$ Middle and High School Rodrigues de Freitas, Porto, Portugal \\ ${ }^{b}$ IFIMUP, University of Porto, Rua do Campo Alegre s/n, 4169-007 Porto, Portugal \\ ${ }^{c}$ Department of Physics and Astronomy, Sciences Education Unit, Faculty of Sciences, University of \\ Porto, Rua do Campo Alegre s/n, 4169-007 Porto, Portugal \\ marcelojrodrigues@sapo.pt ; psimeao@fc.up.pt
}

\begin{abstract}
Video modelling is being used, nowadays, as a tool for teaching and learning several topics in Physics. Most of these topics are related to kinematics. In this work we show how video modelling can be used for demonstrations and experimental teaching in optics, namely the laws of reflection and the well-known Snell's Law of light.

Videos were recorded with a photo camera at 30 frames/s, and analysed with the open source software Tracker. Data collected from several frames was treated with the Data Tool module, and graphs were built to obtain relations between incident, reflected and refraction angles, as well as to determine the refractive index of Perspex. These videos can be freely distributed in the web and explored with students within the classroom, or as a homework assignment to improve student's understanding on specific contents. They present a large didactic potential for teaching basic optics in high school with an interactive methodology.
\end{abstract}

Keywords: laws of optics, video modeling, experimental activity, interactive teaching and learning

\section{INTRODUCTION}

Despite the simplicity of the laws of light reflection and Snell's law, students show some difficulties concerning basic concepts, such as where the reflection angle is measured and what is the normal axis direction. Usually, these laws are studied in schools with optical instruments, which need special care in their manipulation. Moreover, students do not have free access to the laboratories and therefore, there is a limited time for executing the experimental work.

The use videos of real phenomena is a very close approach to reality, has one great advantage for students: the experiment can be seen (and consequently analyzed) as many times as they want. Teachers can record a video or take a photo of one particular experimental detail, or ask students to build their own video recording.

Video modelling can be done with a free analyser software available for didactic purposes, such as Tracker [1-3]. Teachers should define different classroom strategies with these videos, so that they can be used as a demonstration, an exploration or a problem issue, or as we show here as a video based experimental activity (VBEA).

\section{LAWS OF OPTICS}

\subsection{Laws of Reflection}

Figure 1 shows a mirror on the top of a Hartl disc, and two laser beams: one corresponding to the incident light and the other to the reflected one. This figure was taken from a video, available at http://www.fc.up.pt/physletspt/Videos/Reflection_1.mp4, which can be used in class as an experimental activity, or out of class as a homework. 


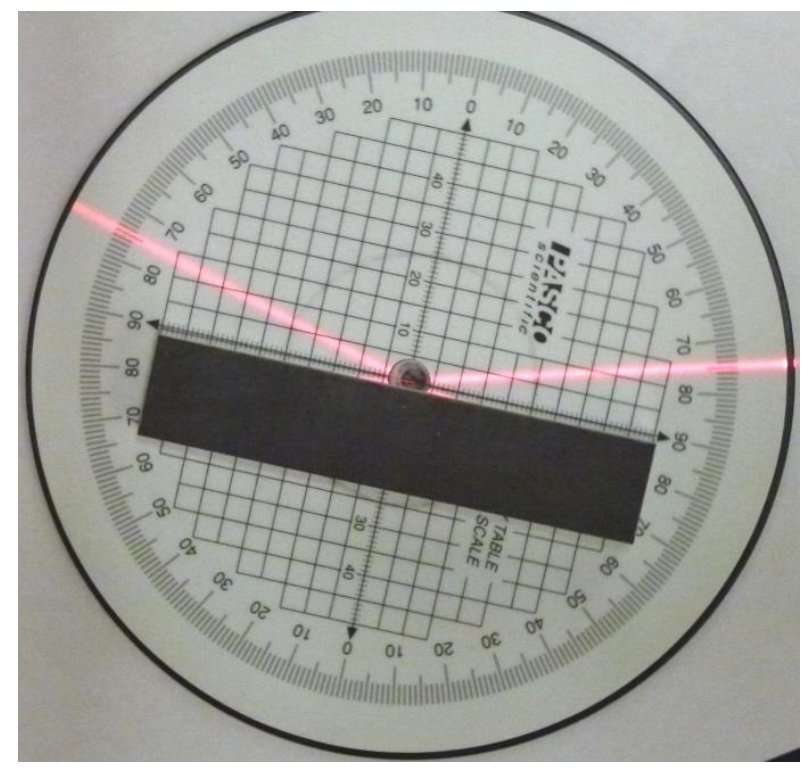

Figure 1: Incident and reflected beams on a flat mirror

To analyze the video and extract experimental data, students have to position the origin of the local referential frame at the ray's incident point, and align one of the referential axis with the mirror's surface while the other axis points to the normal direction (perpendicular to mirror plane). Then they only have to use the protractor tool (either from the Hartl disc, or from Tracker) to measure the angle of the incident and reflected rays against the normal axis (figure 2).

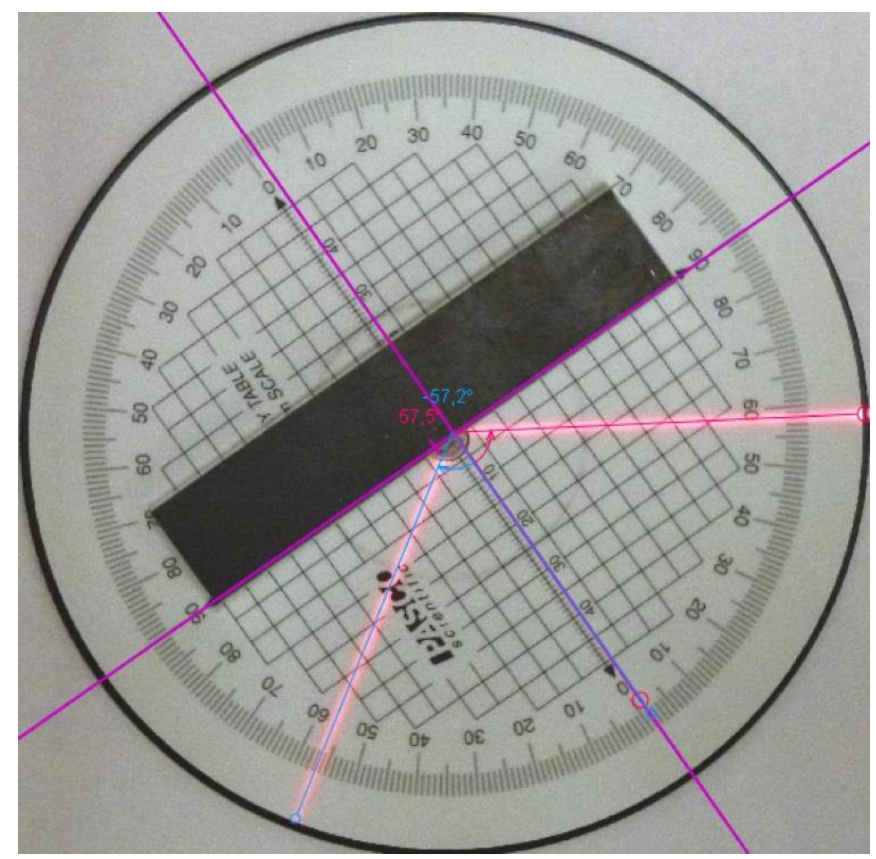

Figure 2: Analysis of one frame of the video with Tracker. The local referential and protractor tools are visualized: the red protractor measures the incident angle while the blue protractor measures the reflected angle. 
As the incident angle changes along the video, students can measure the incident and reflected angles for different frames (don't forgetting to readjust the referential frame at each measurement). This way experimental data is obtained, which will allow them to realize that both incident and reflected angles are equal within an experimental error.

The proposed video based experimental activity (VBEA) has several advantages to students: (1) Manipulation of concepts such as normal axis, incident and reflected rays, incident and reflection angles; (2) Use of the protractor as a tool to measure incident and reflection angles; (3) Understanding the need of a reference frame and; (4) Learn the laws of reflection in a "hands on" point of view.

\subsection{Snell's Law}

The refraction phenomena is more complex than reflection, and the association with quotidian situations like the broken spoon image when submerse in a glass of water, will be better understood if students see what happens with a light ray, at the interface of two transparent media.

The classical classroom experiments of refraction with a glass prism or water, are very important to allow the discussion of this phenomenon, and once again the video analysis can be a good complement to it. By recording a video of the refraction phenomena with a transparent medium, students can use Tracker to analyze it, in a similar way as described above. In our experiment, we have used a semi-circular transparent body that rotates while a red laser beam is placed at a fixed position (figure 3).

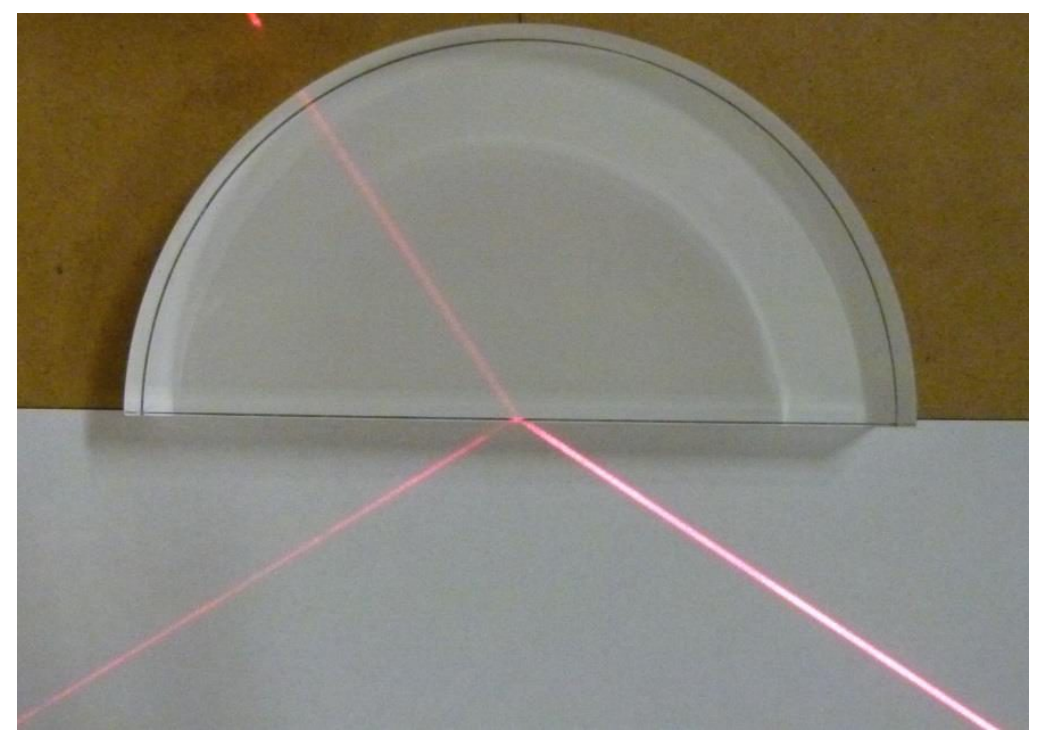

Figure 3: Video frame from our experiment, where a semi-circular body is crossed by a laser beam.

The incident ray is refracted at the flat interface between the body and air. We can observe in figure 3 that the incident angle $\left(\theta_{\mathrm{i}}\right)$ is bigger than the refraction (or transmitted) angle $\left(\theta_{\mathrm{t}}\right)$. Using the protractor tool from Tracker, students can measure these angles for several frames. From Snell's law,

$$
\mathrm{n}_{\text {air }} \sin \theta_{\mathrm{i}}=\mathrm{n}_{\text {body }} \sin \theta_{\mathrm{t}}
$$

and by considering $\mathrm{n}_{\text {air }} \approx 1$, the refraction index of the body can be easily obtained. A statistical treatment of data (linear fit and corresponding standard deviation) can be done from graphic representation of $\sin \theta_{\mathrm{i}} v s . \sin \theta_{\mathrm{t}}$. The value obtained for 
$\mathrm{n}_{\text {body }}$ can be compared to those listed in reference books for acrylic and glass materials, to identify the possible composition of it.

Students can also study the total reflection of light, by providing them a video or a sequence of photos where the incident angle (measured from the transparent body) increases until no light is transmitted, and we can only see the incident and reflected light rays at the flat body-air interface. This will allow teachers to introduce the concept of critical angle, $\theta_{\mathrm{c}}$, and students measure it with the protractor tool. Using, in that case, equation (2),

$$
\mathrm{n}_{\text {body }} \sin \theta_{\mathrm{c}}=1
$$

students can determine $\mathrm{n}_{\text {body }}$ and compare this value to the one previously calculated.

\section{CONCLUSION}

It is usually admitted that video analysis is only engaged to mechanics, in particular kinematics. The new tools incorporated in recent video analysis software like Tracker, enlarges the scientific fields where this software can be used as a teaching and learning tool. In this work we show the advantages of video based experimental activities for teaching concepts in optics. Videos and photos can be used to measure incident, reflected and refracted angles, to study laws of optics and to consolidate contents taught in classroom, as homework tasks or extra-curricular activities. Work is in progress to use Tracker software for teaching physics with videos in a motivating and interactive environment, and results of the respective gains of students' learning will be published elsewhere.

\section{REFERENCES}

[1] Brown, D., "Video Modeling: Combining Dynamic Model Simulations with Traditional Video Analysis", American Association of Physics Teachers (AAPT) Summer Meeting, Edmonton (2008) (available at http://www.cabrillo.edu/ dbrown/tracker/, retrieved in 21/09/2012).

[2] Brown, D., Cox, A.J., "Innovative Uses of Video Analysis”, The Physics Teacher, 47, 145-150. (2009).

[3] Rodrigues, M, Carvalho, P.S., "Teaching Physics with Angry Birds: Exploring the kinematics and dynamics of the game", Physics Education, in the press (2013). 